\title{
LncRNA SNHG3, a potential oncogene in human cancers
}

\author{
Bin $\mathrm{Xu}^{1+}$, Jie $\mathrm{Mei}^{2 \dagger}$, Wei $\mathrm{Ji}^{1 \dagger}$, Zheng Bian ${ }^{1}$, Jiantong Jiao ${ }^{1}$, Jun Sun ${ }^{1 *}\left(\mathrm{D}\right.$ and Junfei Shao ${ }^{1 *}$
}

\begin{abstract}
Long noncoding RNAs (IncRNAs) are composed of > 200 nucleotides; they lack the ability to encode proteins but play important roles in a variety of human tumors. A large number of studies have shown that dysregulated expression of IncRNAs is related to tumor oncogenesis and progression. Emerging evidence shows that SNHG3 is a novel oncogenic IncRNA that is abnormally expressed in various tumors, including osteosarcoma, liver cancer, lung cancer, etc. SNHG3 primarily competes as a competitive endogenous RNA (ceRNA) that targets tumor suppressor microRNAs (miRNAs) and ceRNA mechanisms that regulate biological processes of tumors. In addition, abnormal expression of SNHG3 is significantly correlated with patient clinical features. Upregulation of SNHG3 contributes to biological functions, including tumor cell proliferation, migration, invasion and EMT. Therefore, SNHG3 may represent a potential diagnostic and prognostic biomarker, as well as a novel therapeutic target.
\end{abstract}

Keywords: LncRNA, SNHG3, Cancer, Biomarker

\section{Background}

Recent studies have shown that cancer remains a global problem [1-3]. Numerous studies have shown that noncoding RNAs (ncRNAs) do not encode proteins but exert their function through various mechanisms, which are important aspects of cellular regulation [4-6]. Noncoding RNAs can be categorized according to length into small ncRNAs ( $<200$ nucleotides) and long ncRNAs ( $>200$ nucleotides, lncRNAs). LncRNAs are differentially classified based on place of their origin on the genome, including long intergenic noncoding RNA, natural antisense transcripts, circular RNA, pseudogene transcripts, transcribed ultraconserved regions, and telomerase RNA components [7-9].

LncRNAs are primarily transcribed by RNA polymerase II but are not translated into proteins $[10,11]$. A growing number of studies have shown that lncRNAs play an important role in a variety of biological processes, including

\footnotetext{
*Correspondence: wxrmsj@163.com; wxrmyysj@163.com

${ }^{\dagger} \mathrm{Bin}$ Xu, Mei Jie and Wei Ji contributed equally to this work

${ }^{1}$ Department of Neurosurgery, Wuxi People's Hospital Affiliated

to Nanjing Medical University, No. 299 Qing Yang Road, Wuxi 214023, Jiangsu, China

Full list of author information is available at the end of the article
}

transcription, translation, and epigenetics. LncRNAs may act as tumor suppressor genes and oncogenes, affecting processes like cell proliferation, apoptosis, differentiation, invasion, migration, and suppression of immune responses. In addition, several lncRNAs exhibit cell- and tissue-specific expression patterns. Therefore, lncRNAs could be used as molecular markers for the early diagnosis of tumors and as novel targets for tumor treatment [12-18].

The small nuclear protein RNA host gene 3 (SNHG3) belongs to a group of long noncoding RNAs that are associated with multiple cancers and are dysregulated in multiple cancers. Recent studies have shown that SNHG3 expression is higher in many tumors compared to normal tissues. Furthermore, overexpression of SNHG3 significantly promotes tumor proliferation, migration and invasion, indicating that SNHG3 is a carcinogenic lncRNA. In addition, SNGH3 was previously identified by Honjo et al. as a nonimmunoglobulin target locus of the activation-induced cytidine deaminase (AID) pathway [19]. Lu et al. [20] found that SNHG3 is essential for mouse embryonic stem cell (mESC) self-renewal and pluripotency, as well as mouse early embryo development. This review summarizes research progress on the abnormal

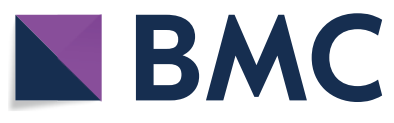

c) The Author(s) 2020. This article is licensed under a Creative Commons Attribution 4.0 International License, which permits use, sharing, adaptation, distribution and reproduction in any medium or format, as long as you give appropriate credit to the original author(s) and the source, provide a link to the Creative Commons licence, and indicate if changes were made. The images or other third party material in this article are included in the article's Creative Commons licence, unless indicated otherwise in a credit line to the material. If material is not included in the article's Creative Commons licence and your intended use is not permitted by statutory regulation or exceeds the permitted use, you will need to obtain permission directly from the copyright holder. To view a copy of this licence, visit http://creativeco mmons.org/licenses/by/4.0/. The Creative Commons Public Domain Dedication waiver (http://creativecommons.org/publicdomain/ zero/1.0/) applies to the data made available in this article, unless otherwise stated in a credit line to the data. 
expression, functions, molecular mechanisms and clinical significance of SNHG3 in tumorigenesis and cancer progression (Tables 1, 2).

\section{Characterization of SNHG3}

Small nucleolar RNAs (snoRNAs) are primarily transcribed from protein coding gene clusters or other ncRNA coding genes. Some snoRNA genes without coding ability still contain introns and exons in their sequences, but snoRNAs are only produced by introns. If full-length transcripts, including exons, are retained, they will function as IncRNAs, called small nucleolar RNA host genes (SNHGs) [21]. SNHG3 is an oncogenic lncRNA that produces SNORD17. SNHG3 is not only found in the nucleus but also in the cytoplasm [22] (Fig. 1). SNHG3 is located on chromosome 1p35.3 and

Table 1 Functional characterization of SNHG3 in various cancers

\begin{tabular}{|c|c|c|c|c|c|}
\hline Cancer type & Expression & Role & Biological functions & Related genes & Refs. \\
\hline Breast cancer & Up & Oncogenic & $\begin{array}{l}\text { Promotes cell proliferation, migration } \\
\text { and invasion }\end{array}$ & $\begin{array}{l}\text { miR-384/HDGF } \\
\text { miR-330-5p/PKM } \\
\text { miR-326/ITGA5, Vav2/Rac1 }\end{array}$ & {$[31-33]$} \\
\hline Osteosarcoma & Up & Oncogenic & $\begin{array}{l}\text { Promotes cell proliferation, migra- } \\
\text { tion, invasion and EMT }\end{array}$ & $\begin{array}{l}\text { miR-151a-3p/RAB22A, miR-196a-5p/ } \\
\text { HOXC8 }\end{array}$ & {$[25,38]$} \\
\hline Glioma & Up & Oncogenic & $\begin{array}{l}\text { Promores cell proliferation, inhib- } \\
\text { its apoptosis, and regulates cell } \\
\text { cycle }\end{array}$ & KLF2, p21, EZH2 & {$[24]$} \\
\hline Laryngeal cancer & UP & Oncogenic & $\begin{array}{l}\text { Promotes cell migration, invasion } \\
\text { and cell viability, glycolysis }\end{array}$ & $\begin{array}{l}\text { miR-384/WEE1, MMP2, MMP9 } \\
\text { miR-340-5p/YAP1, Wnt/ } \beta \text {-catenin }\end{array}$ & {$[44,45]$} \\
\hline Gastric cancer & Up & Oncogenic & $\begin{array}{l}\text { Promotes cell proliferation, migra- } \\
\text { tion, invasion and cell viability }\end{array}$ & EZH2, MED18 & {$[23]$} \\
\hline Colorectal cancer & Up & Oncogenic & $\begin{array}{l}\text { Promotes cell proliferation, migra- } \\
\text { tion, invasion and cell viability }\end{array}$ & miR-182-5p/c-Myc, miR-539/RUNX2 & {$[53,54]$} \\
\hline Renal cell carcinoma & Up & Oncogenic & $\begin{array}{l}\text { Promotes cell proliferation, cell } \\
\text { viability, migration, invasion and } \\
\text { EMT and inhibits cell apoptosis }\end{array}$ & $\begin{array}{l}\text { miR-139-5p/TOP2A, CDK6, Bax, Bcl-2, } \\
\text { N-cadherin, E-cadherin, Vimentin }\end{array}$ & {$[57]$} \\
\hline Hepatocellular carcinomac & Up & Oncogenic & $\begin{array}{l}\text { Promotes cell proliferation, apopto- } \\
\text { sis, migration, invasion, EMT and } \\
\text { sorafenib resistance }\end{array}$ & $\begin{array}{l}\text { miR-139-5p/BMI1, miR-326/SMAD3/ } \\
\text { ZEB1, miR-128/CD151/AKT/PI3K, } \\
\text { N-cadherin, E-cadherin, Vimentin, } \\
\text { Snail }\end{array}$ & {$[60-63]$} \\
\hline Lung cancer & Up & Oncogenic & $\begin{array}{l}\text { Promotes cell proliferation, migra- } \\
\text { tion and EMT, inhibits cell apopto- } \\
\text { sis and regulates cell cycle }\end{array}$ & TGF- $\beta$, IL-6/JAK2/STAT3 & {$[66,67]$} \\
\hline Acute myeloid leukemia & Up & Oncogenic & $\begin{array}{l}\text { Promotes cell proliferation and } \\
\text { inhibits cell apoptosis }\end{array}$ & miR-758-3p/SRGN & {$[70,71]$} \\
\hline Epithelial ovarian cancer & Up & Oncogenic & $\begin{array}{l}\text { Promotes cell proliferation, invasion } \\
\text { and glycolysis }\end{array}$ & $\begin{array}{l}\text { CyclinD1, CDK1, MMP9, MMP3, } \\
\text { GSK3 } \beta / \beta \text {-catenin, EIF4A3-mRNA }\end{array}$ & {$[26,74]$} \\
\hline \multirow[t]{2}{*}{ Papillary thyroid carcinoma } & Up & Oncogenic & $\begin{array}{l}\text { Promotes cell proliferation, migration } \\
\text { and invasion }\end{array}$ & miR-214-3p/PSMD10 & {$[76]$} \\
\hline & Down & $\begin{array}{l}\text { Tumor Suppres- } \\
\text { sive }\end{array}$ & $\begin{array}{l}\text { Promotes cell proliferation, migration } \\
\text { and invasion }\end{array}$ & AKT/mTOR/ERK & {$[77]$} \\
\hline Prostate cancer & UP & Oncogenic & $\begin{array}{l}\text { Promotes cell proliferation, migra- } \\
\text { tion, invasion, EMT process } \\
\text { and inhibits cell apoptosis }\end{array}$ & miR-577/SMURF1 & {$[78]$} \\
\hline Bladder cancer & Up & Oncogenic & $\begin{array}{l}\text { Promotes cell proliferation, migra- } \\
\text { tion, invasion and EMT }\end{array}$ & miR-515-5p/GINS2 & {$[79]$} \\
\hline Oral squamous cell carcinoma & Up & Oncogenic & $\begin{array}{l}\text { Promotes cell migration, invasion } \\
\text { and proliferation }\end{array}$ & ELAVL1/NFYC, Wnt/ $\beta$-catenin & {$[80]$} \\
\hline
\end{tabular}

EMT epithelial-mesenchymal transition, HDGF hepatoma-derived growth factor, PKM pyruvate kinase M1/M2, ITGA5 integrin a5, RAB22A Ras-related proteins 22a, HOXC8 homeobox C8, KLF2 kruppel like factor 2, p21 p21 protein, EZH2 Enhancer of zeste homolog 2, WEE1 wee1-like protein kinase, MMP2 matrix metalloproteinase-2, MMP9 matrix metalloproteinase-9, YAP1 yes-associated protein 1, MED18 mediator subunit 18, RUNX2 runt-related transcription factor 2, TOP2A the expression of topoisomerase Ila, CDK6 cyclin-dependent kinase $6, N$-cadherin neural-cadherin, $E$-cadherin epithelial-cadherin, $B M I 1 \mathrm{~B}$ lymphoma Mo-MLV insertion region 1, SMAD3 Sma and Mad Related Family 3, ZEB1 zinc finger E-box binding homeobox 1, PI3K phosphoinositide-3 kinase, TGF- $\beta$ transforming growth factor- $\beta$, IL-6 the interleukin-6, JAK2 Janus kinase 2, STAT3 signal transducer and activator of transcription 3, SRGN serglycin, GSK3 $\beta$ glycogen synthase kinase 3 beta, EIF4A3 eukaryotic translation initiation factor 4A3, SMURF1 Smad Ubiquitin Regulatory Factor 1, GINS2 GINS complex subunit 2, PSMD10 proteasome 26 S subunit non-ATPase 10, ELAVL1 ELAV like RNA-binding protein 1, NFYC nuclear transcription factor Y subunit gamma 
Table 2 Clinical significance of SNHG3 in diverse cancers

\begin{tabular}{|c|c|c|}
\hline Cancer types & Overexpression of SNHG3 and clinical features & Refs. \\
\hline Breast cancer & ER status, HER-2 status, tumor size, histological grade, lymph node metastasis and advanced TNM stage & {$[31-33]$} \\
\hline Osteosarcoma & Tumor size and poor overall survival & {$[25,38]$} \\
\hline Glioma & Poor overall survival & {$[24]$} \\
\hline Laryngeal cancer & No description & {$[44,45]$} \\
\hline Gastric cancer & Poor prognosis and lymph node metastasis & {$[23]$} \\
\hline Colorectal cancer & Distant metastasis, advanced TNM stage and poor prognosis & {$[53,54]$} \\
\hline Renal cell carcinoma & Tumor size, distant metastasis, T stage, pathological TNM stage, histologic grade and poor prognosis & {$[57]$} \\
\hline Hepatocellular carcinoma & Tumor size, histologic grade and poor prognosis & {$[60-63]$} \\
\hline Lung cancer & Tumor size, TNM stage, lymph node migration and poor prognosis & {$[66,67]$} \\
\hline Leukemia & Poor prognosis & {$[70,71]$} \\
\hline Ovarian cancer & Poor prognosis, lymph node metastasis, FIGO stage and drug resistance & {$[26,74]$} \\
\hline Papillary thyroid carcinoma & Lymph node metastasis, tumor node metastasis stages and poor prognosis & {$[76,77]$} \\
\hline Prostate cancer & No description & {$[78]$} \\
\hline Bladder cancer & Tumor size, metastasis and poor clinical prognosis & {$[79]$} \\
\hline Oral squamous cell carcinoma & No description & {$[80]$} \\
\hline
\end{tabular}

TNM tumor node metastasis, ER estrogen receptor, HER-2 human epidermal growth factor receptor-2, FIGO international federation of gynecology and obstetrics

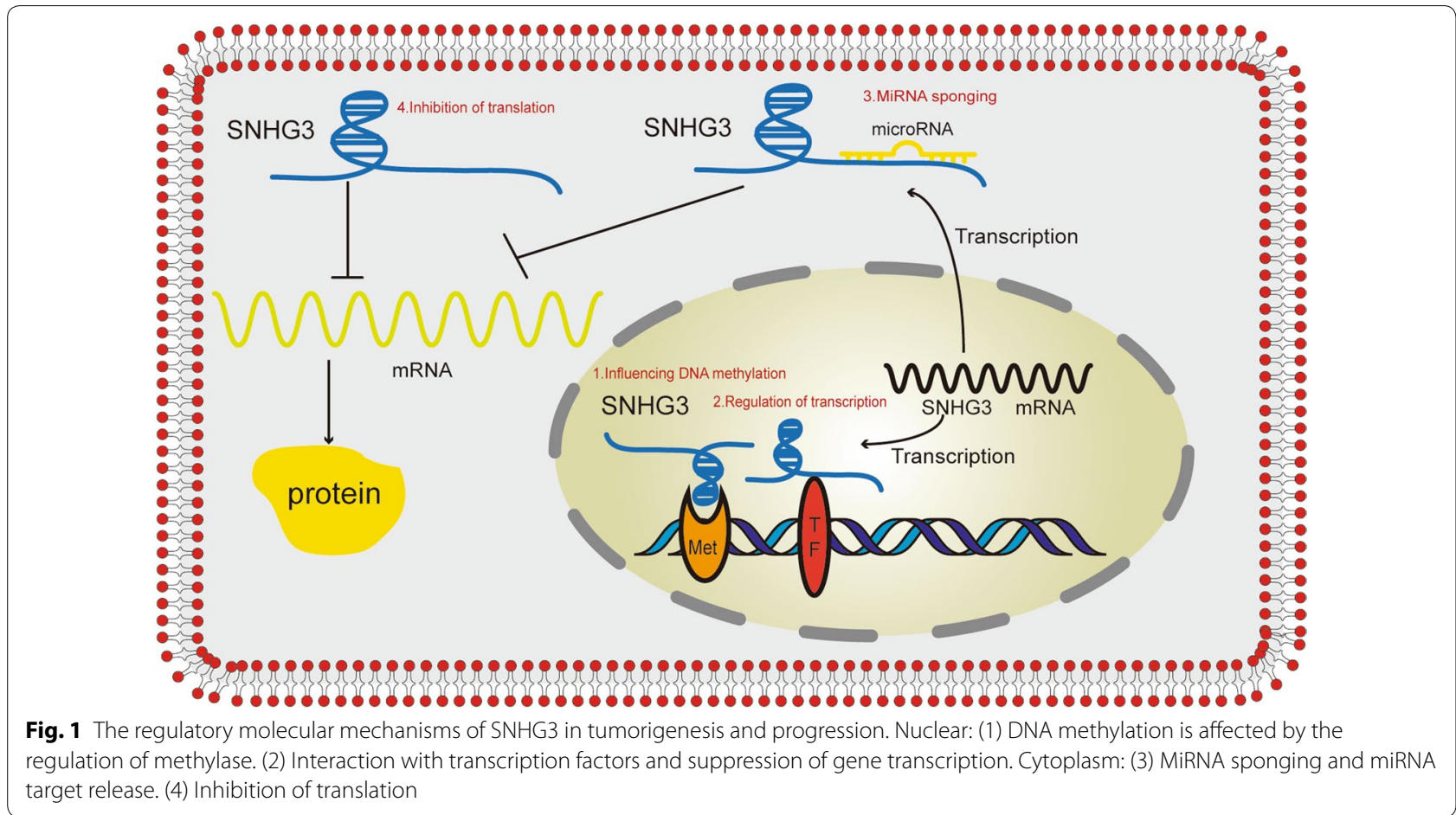

has four exons that comprise $4950 \mathrm{bp}$. The Human Protein Atlas (HPA) project RNA-seq normal tissue results suggest that SNHG3 is more highly expressed in the bone marrow and appendix than in other human tissues. In the SNHG3 literature, we identified four major molecular mechanisms of action with different subcellular localizations. Nuclear: (1) DNA methylation is affected by the regulation of methylase. For instance, SNHG3 promotes gastric cancer progression through regulating neighboring mediator subunit 18 (MED18) gene methylation by binding to enhancer of zeste homolog 2 (EZH2) [23]. (2) Interaction with transcription factors and suppression of gene transcription. For instance, SNHG3 enhances the malignant behaviors of glioma through silencing 
Kruppel-like factor 2 (KLF2) and p21 protein (p21) via recruiting EZH2 to the promoter of KLF2 and p21 [24]. Cytoplasm: (3) MiRNA sponging and miRNA target release. For instance, SNHG3 sponges miR-151a-3p as a ceRNA to upregulate Ras-related protein 22a (Rab22a) expression, thus increasing cell migration and invasion of osteosarcoma cells [25]. (4) Inhibition of translation. For instance, SNHG3 is related to energy metabolism by regulating eukaryotic translation initiation factor 4A3 (EIF4A3) mRNA in ovarian cancer (OC) [26]. A growing number of studies have shown that SNHG3 plays a crucial role in the development and prognosis of a variety of malignancies.SNHG3 may represent a valuable prognostic biomarker and therapeutic target in various cancers.

\section{LncRNA SNHG3 dysregulation in human cancers LncRNA SNHG3 in breast cancer}

Breast cancer is the most common cancer among American women and the second leading cause of cancer death in women, second only to lung cancer [27, 28]. Metastasis of cancer cells is one of the leading causes of death in breast cancer patients [29]. Although significant progress has been made in the diagnosis and treatment of breast cancer, the mortality rate of breast cancer patients remains high [30]. Therefore, further study of the molecular mechanism of breast cancer is crucial. Ma et al. [31] examined SNHG3 expression in 60 pairs of breast cancer tissues and observed remarkable upregulation of SNHG3 expression in breast cancer tissues and cells. In addition, its overexpression was significantly associated with histological grade, lymph node metastasis, advanced tumor node metastasis (TNM) stage, and estrogen receptor (ER) and human epidermal growth factor receptor 2 (Her-2) status. Functionally, SNHG3 knockdown hindered breast cancer cell proliferation, migration and invasion in vitro and in vivo. Moreover, SNHG3 expression is positively correlated with hepatoma-derived growth factor (HDGF) expression. Furthermore, SNHG3 acts as a competing endogenous RNA by directly binding to miR-384 in a sequence-specific manner. Meanwhile, HDGF is also a target gene of miR-384 and is regulated by SNHG3. In other words, SNHG3 functions as an endogenous sponge by sequestering miR-384, thus abolishing the miRNA-induced repressive effect on HDGF. Yan et al. [32] reported that SNHG3 uses a miR-330-5p sponge to positively regulate pyruvate kinase M1/M2 (PKM) expression, inhibit mitochondrial oxidative phosphorylation, and enhance breast cancer cell proliferation. Wang et al. [33] found that SNHG3 silencing enhances apoptosis in triple-negative breast cancer (TNBC) cells through the miR-326/integrin $\alpha 5$ (ITGA5) axis and inhibits cell viability, migration, invasion and the Vav2/Rac1 signaling pathway. These results demonstrated that SNHG3 acts as an oncogenic lncRNA in breast cancer, which may represent a potential diagnostic biomarker or a novel therapeutic target for cancers [34].

\section{LncRNA SNHG3 in osteosarcoma}

Osteosarcoma (OS) is the most common malignant bone tumor, primarily affecting adolescents. OS frequently affects long bones and is prone to lung metastasis [35]. Although treatment of OS has made great progress, the prognosis of OS patients is still poor due to distant metastasis [36]. Therefore, novel and effective prognostic biomarkers and therapeutic targets for the disease must be identified [37]. Zheng et al. [25] found that expression of SNHG3 and Rab22a was upregulated in 54 OS tissues and OS cell lines (Saos2, MG63, U2OS and HOS) compared to paired normal tissues and normal osteoblasts, while miRNA-151a-3p was expressed at low levels. Overexpression of SNHG3 accelerates the invasive and migratory potentials of OS cells. SNHG3 overexpression in MG63 cells markedly enhanced their phenotype, and SNHG3 knockdown in U2OS cells inhibited their phenotype. SNHG3 upregulates Rab22a expression, and SNHG3 binds to Rab22a and miRNA-151a-3p. A previous study demonstrated that the SNHG3/miRNA151a-3p/Rab22a axis regulates invasion and migration of osteosarcoma. Chen et al. [38] also confirmed that expression of SNHG3 was upregulated in both OS tissues and OS cell lines compared to paired normal tissues and normal osteoblasts. Overexpression of SNHG3 markedly increases cell viability and colony number. SNHG3 acts as a sponge of miRNA-196a-5p in OS cells. These results indicated that SNHG3 promotes the growth of OS cells by sponging the miRNA-196a-5p/homeobox c8 (HOXC8) axis, providing a potential marker in OS patients.

\section{LncRNA SNHG3 in glioma}

Glioma is one of the most common major brain tumors in adults and one of the most important malignant tumors in humans [1]. Although surgery, chemotherapy, radiotherapy and other treatments have made great progress, the clinical prognosis of glioma patients remains poor [39]. Therefore, it is important to understand the molecular mechanisms underlying the development of gliomas [40]. Fei et al. [24] uncovered high expression levels of SNHG3 in glioma tissues and glioma cell lines compared to nontumor tissues and normal cells. Furthermore, upregulated SNHG3 suggests a poor prognosis in patients with glioma. Silencing of SNHG3 inhibits cell proliferation by triggering apoptosis and cell cycle arrest. After SNHG3 was overexpressed in U251 cells, the opposite results were observed. In addition, KLF2 and p21 were downregulated in glioma tissues. SNHG3 was 
negatively correlated with p21 and KLF2. These experiments revealed that SNHG3 accelerates the malignancy of glioma by inhibiting transcription of KLF2 and p21. Generally, it is believed that SNHG3 may be an oncogene in glioma and may serve as a potential prognostic biomarker and therapeutic target for glioma.

\section{LncRNA SNHG3 in laryngeal cancer}

Laryngeal cancer (LC) is a common malignant tumor of the head and neck, whose main pathological type is squamous cell carcinoma. The primary risk factors for LC include smoking, alcohol consumption, environmental factors, radioactivity, viral infections, and trace element deficiency [41]. Current treatment strategies include radiation therapy and surgical removal of tumors [42]. Therefore, early diagnosis and treatment are essential for maintaining laryngeal function and reducing postoperative complications [43]. Wang et al. [44] determined that expression of SNHG3 was upregulated in LC tissues and cell lines compared to normal tissues and cell lines. Moreover, downregulation of SNHG3 significantly depressed migration and invasion capacities, as well as matrix metalloproteinase-2 (MMP2) and matrix metalloproteinase-9 (MMP9) protein levels. SNHG3 negatively regulates miR-384, improves wee1-like protein kinase (WEE1) expression, and promotes LC cell migration and invasion. Furthermore, SNHG3 acts as a ceRNA by directly binding to miR-384 to increase WEE1 expression. In summary, these results indicated that SNHG3 regulates the migration and invasion of LC cells via the miR-384/WEE1 axis. Kang et al. [45] found that knock down of SNHG3 reduced the growth of LSCC xenograft tumors by regulating the miR-340-5p/yes-associated protein 1 (YAP1) axis and $\mathrm{Wnt} / \beta$-catenin pathway. As mentioned above, SNHG3 may represent a potential therapeutic target for LC patients.

\section{LncRNA SNHG3 in gastric cancer}

Gastric cancer (GC) is one of the most common malignancies in humans [46]. The most common cause of gastric cancer is Helicobacter pylori $(H p)$ infection, accounting for more than half of the total incidence [47, 48]. GC treatment primarily includes surgery, chemotherapy, radiotherapy and targeted therapy [49]. Early diagnosis helps patients who are in the early stages of disease progression. There is ample evidence that lncRNAs play an indispensable role in promoting tumor or antitumor effects in human malignancies [50]. Xuan et al. [23] observed apparent overexpression of SNHG3 in cancer cell lines and tissues in comparison with normal cells and tissues. High SNHG3 in GC also predicted poor prognosis. SNHG3 promoted the proliferation of gastric cancer cells both in vitro and in vivo. Conversely, downregulation of SNHG3 inhibited the migration, invasion and metastasis of GC cells both in vitro and in vivo. SNHG3 and EZH2 simultaneously bind to MED18 promoter, and SNHG3 exerts apparent regulation of neighboring gene MED18 transcription in GC cells. In conclusion, their results demonstrated that MED18 exhibits antitumor activity in GC cells by inhibiting cell proliferation, migration and invasion. The anticancer effect of MED18 occurs downstream of SNHG3 signaling in gastric cancer, which may imply its widespread mode of action in other cancers, and deserves further study.

\section{LncRNA SNHG3 in colorectal cancer}

Colorectal cancer (CRC) is the third most common malignancy in the world with high morbidity and mortality rates [46]. Although advanced treatments exist, including surgical resection, chemotherapy, radiation, the 5-year and 10-year survival rates in CRC patients are still unsatisfactory at $65 \%$ and $58 \%$, respectively [51]. In summary, a better understanding of the precise molecular mechanisms that promote the development of CRC will be beneficial to help CRC patients and to identify new diagnostic methods or treatment strategies [52]. Huang et al. [53] showed that SNHG3 was highly expressed in CRC tumor tissues compared to adjacent normal tissues. Overexpression of SNHG3 significantly increased the proliferative ability of CRC cells. Consistently, SNHG3 downregulation inhibited CRC cell proliferation. Their results indicate that SNHG3 and c-Myc share the same miRNA-responsive element with miR-182-5p and promote CRC progression in a ceRNA manner. Wen et al. [54] revealed that knockdown of the SNHG3 gene significantly reduces growth and metastasis of CRC. Mechanistically, SNHG3 acts as the miRNA's ceRNA to bind miR-539, thereby regulating the expression of its target gene, runt-related transcription factor 2 (RUNX2), and promoting the occurrence and development of CRC. Upregulation of SNHG3 is correlated with poor prognosis, indicating that it may be an important biomarker of CRC.

\section{LncRNA SNHG3 in renal cell carcinoma}

Renal cell carcinoma (RCC) accounts for $5 \%$ of adult malignancies. It is estimated that there were approximately 73,820 new cases and 14,770 renal cell carcinoma deaths in the United States in 2019 [46]. Conventional radiotherapy and chemotherapy are not ideal, and surgery is the primary method for treating renal cell carcinoma. In recent years, targeted therapy has been found to improve patient prognosis, but the emergence of drug resistance has hindered the development of targeted 
drugs. Therefore, it is crucial to further identify new therapeutic targets for RCC [55, 56]. Zhang et al. [57] found that SNHG3 acts as a ceRNA of mir-139-5p and inhibits the activity of mir-139-5p. Expression of topoisomerase II $\alpha$ (TOP2A), a target of miR-139-5p, was increased, which ultimately promoted proliferation and metastasis of renal cancer cells. These results confirmed that the SNHG3/miR-139-5p/TOP2A axis plays a crucial role in renal cell carcinoma and may represent a key target for diagnosis and treatment.

\section{LncRNA SNHG3 in hepatocellular carcinoma}

Hepatocellular carcinoma (HCC) is one of the major malignancies in the world [58]. Over the years, there have been several advances in the diagnosis and treatment of HCC. Due to its high recurrence rate and high distant metastasis rate, the overall survival (OS) and 5-year survival rate of HCC patients are still low. Therefore, it is very urgent to further study the pathogenesis of HCC and discover new targets for diagnosis and treatment [59]. Wu et al. [60] reported that the lncSNHG3/ miR-139-5p/B lymphoma Mo-MLV insertion region 1 (BMI1) axis plays an important role in cell proliferation, migration, and invasion in HCC. Zhao et al. [61] showed that overexpression of SNHG3 promotes proliferation, migration and epithelial-mesenchymal transition (EMT) of HCC and inhibits apoptosis, while knockdown of SNHG3 plays the opposite role. The proposed mechanism suggested that SNHG3 may act as a ceRNA of miR326, increasing expression levels of Sma and mad related family 3 (SMAD3) and zinc finger E-box binding homeobox 1 (ZEB1). In conclusion, SNHG3 promotes hepatocellular tumorigenesis via the miR-326/SMAD3/ZEB1 axis. Zhang et al. [62] found that expression of SNHG3 in highly metastatic HCC cells was significantly higher than in low metastatic HCC cells. Functionally, high expression of SNHG3 promotes cell invasion, EMT and sorafenib resistance. In addition, SNHG3 acts as a ceRNA and induces EMT in hepatoma cells via the miR128/ CD151 signaling pathway. Zhang et al. [63] reported that elevated expression of SNHG3 in liver cancer patients was associated with malignant status and poor prognosis. Taken together, their data suggest that SNHG3 may represent a new therapeutic target and a biomarker for predicting HCC response to sorafenib [64].

\section{LncRNA SNHG3 in lung cancer}

Lung cancer is a major, worldwide, malignant and deadly tumor. Clinically, methods for treating lung cancer include radiotherapy, chemotherapy, surgery, etc. However, lung cancer patients have poor prognosis, and the 5 -year survival rate is only $16 \%[1,46]$. To further elucidate the molecular mechanism of lung adenocarcinoma, we are looking for new diagnostic and therapeutic targets for the treatment of lung adenocarcinoma [65]. Shi et al. showed that expression of SNHG3 in non-smallcell lung cancer (NSCLC) tissues and cells was higher than in normal tissues and cell lines. In addition, NSCLC patients with high SNHG3 expression had a low overall survival rate. Functionally, high expression of SNHG3 significantly promotes cell proliferation and migration. Mechanistically, SNHG3 is activated by E2F transcription factor 1 (E2F1), subsequently promoting proliferation and migration of NSCLC by activating the transforming growth factor- $\beta$ (TGF- $\beta$ ), interleukin-6 (IL-6)/janus-activated kinase 2 (JAK2)/signal transducer activator of transcription 3 (STAT3) pathways [66]. Liang et al. [67] also confirmed that overexpression of SNHG3 promotes lung adenocarcinoma cell proliferation and the cell cycle, as well as inhibiting apoptosis. Therefore, SNHG3 may be a potential new target for the treatment and prognosis of lung adenocarcinoma.

\section{LncRNA SNHG3 in leukemia}

Leukemia is a relatively rare cancer with a median age of approximately 60 years [68]. A considerable number of studies have shown that acute myeloid leukemia (AML) is a highly heterogeneous collection of diseases that may be genetically altered in terms of cell morphology, cytochemistry, immunophenotype, cytogenetics, and molecular abnormalities [69]. Therefore, it is urgent to further study the mechanism of leukemia occurrence and development to identify new biomarkers and treatment strategies. Peng et al. [70] revealed that silencing SNHG3 inhibits cell proliferation and induces apoptosis. In addition, downregulation of SNHG3 significantly reduced the expression of SRGN in AML cells. Mechanistically, we found that SNHG3 regulates the expression of serglycin (SRGN) by competitively binding to miR-758-3p. In other words, SNHG3 promotes the growth of acute myeloid leukemia cells by regulating the miR-758-3p/SRGN axis, providing a new therapeutic direction for the treatment of AML. According to the dynamic network biomarker (DNB) standard, some dysregulated lncRNA-associated ceRNA network biomarkers for chronic myeloid leukemia (CML) have been identified and analyzed. Xu et al. [71] found that SNHG3 is an effective CML biomarker that helps patients obtain timely treatment and reduces CML mortality.

\section{LncRNA SNHG3 in epithelial ovarian cancer}

Epithelial ovarian cancer (EOC) is the deadliest gynecological malignancy. Due to the asymptomatic and concealed nature of ovarian cancer, EOC patients are often clinically advanced when ultimately diagnosed, which poses a huge obstacle to clinical diagnosis and treatment 
[72]. Therefore, it is urgent to further elucidate the molecular mechanism of ovarian cancer to identify molecules for early diagnosis. Li et al. [73] found that lncRNA SNHG3 regulates energy metabolism in ovarian cancer as shown by mitochondrial proteomic analysis. Hong et al. [74] showed that expression of SNHG3 in ovarian cancer tissues was significantly higher than in adjacent normal tissues. In addition, SNHG3 was highly positively associated with FIGO stage, lymph node metastasis, and poor prognosis. Overexpression of SNHG3 promoted the proliferation and invasion of ovarian cancer cells, resulting in a significant downregulation of CyclinD1, CDK1, MMP9 and MMP3. Results also demonstrated that regulation of the glycogen synthase kinase 3 beta (GSK3 $\beta$ )/ $\beta$ catenin signaling pathway promotes the development of ovarian cancer. Li et al. [26] uncovered that ivermectin inhibits $\mathrm{OC}$ migration by potentially targeting the lncRNA-EIF4A3-mRNA pathway, establishing an effective prognostic model. These findings indicate that OC treatment and parents' postoperative pain measurement (PPPM) prognostic assessment have practical significance. In conclusion, SNHG3 may serve as a novel target for the diagnosis and treatment of ovarian cancer.

\section{LncRNA SNHG3 in papillary thyroid carcinoma}

Papillary thyroid cancer (PTC) is the most common type of thyroid cancer and ranks ninth in global cancer incidence [46]. Although most PTCs show good prognosis, a small number of patients experience aggressive and refractory disease [75]. Therefore, it is urgent to understand the molecular mechanism of PTC tumor occurrence and progression to further improve the cure rate and survival rate in patients. Sui et al. [76] showed that SNHG3 can be used as the ceRNA of this miRNA to bind to miR-214-3p, actively regulating expression of the proteasome $26 \mathrm{~S}$ subunit non-ATPase 10 (PSMD10) and significantly reducing PTC in vitro cell migration, invasion, proliferation and colony formation. These results suggest that SNHG3 may be a diagnostic and therapeutic target for PTC. Duan et al. [77] found that lncRNA SNHG3 was significantly downregulated in PTC tissues and cell lines. Functional studies have shown that SNHG3 deletion promotes the proliferation, migration and invasion of PTC cells. Further mechanistic analysis showed that knockout of SNHG3 promotes the occurrence of PTC tumors both in vivo and in vitro by activating the $\mathrm{AKT} / \mathrm{mTOR} / \mathrm{ERK}$ pathway. Of note, SNHG3 may become a promising candidate for PTC targeted therapy.

\section{SNHG3 in other cancers}

$\mathrm{Li}$ et al. [78] reported that SNHG3 is highly expressed in prostate cancer cell lines. SNHG3 knockdown inhibits prostate cancer cell proliferation, migration, and
EMT processes, promoting cell growth. Mechanistically, SNHG3 endogenously absorbs miR-577, thereby positively expressing smad ubiquitin regulatory factor 1 (SMURF1) and promoting the development of prostate cancer. We experimentally discovered demonstrated that the carcinogenic effects of SNHG3 and SMURF1 in prostate cancer may provide new ideas for biomarkers of prostate cancer. Dai et al. [79] identified that lncRNA SNHG3 is upregulated in bladder cancer tissues, and lncRNA SNHG3 knockdown inhibits bladder cancer cell proliferation, migration, invasion and EMT processes both in vitro and in vivo. In addition, under the mechanism of ceRNA, SNHG3 sponges miR515-5p to escalate expression of the GINS complex subunit 2 (GINS2). Therefore, research shows that lncRNA SNHG3 may become a new diagnostic and treatment target for bladder cancer. Liu et al. [80] confirmed that SNHG3 participates in the progression of oral squamous cell carcinoma (OSCC) by regulating the ELAV-like RNA-binding protein 1 (ELAVL1)/nuclear transcription factor $\mathrm{Y}$ subunit gamma (NFYC) axis and the Wnt/ $\beta$-catenin pathway. In summary, SNHG3 may represent a new biomarker for OSCC.

\section{Perspectives in clinical practice \\ Diagnostic and prognostic value of SNHG3 in cancer} assessment

Pancancer analysis reveals that long-chain noncoding RNAs have potential diagnostic and prognostic value in a variety of cancers [81]. With the continuous development of RNA sequencing, gene microarrays and high-throughput sequencing, we recognize that lncRNAs can act as tumor suppressors and oncogenes, playing key roles in regulating tumor formation and development [82]. With the study of the molecular mechanism of lncRNAs in tumors, lncRNAs may become new tumor biomarkers for the diagnosis and treatment of cancer targets [83]. Yang et al. [84] used RNA-seq and survival data in the TCGA database to analyze the expression profiles of 20 SNHGs and discussed their prognostic value in clear cell renal cell carcinoma (ccRCC). Results of the study indicated that SNHG3 and SNHG15 act as diagnostic markers and indicators and may have great clinical value to evaluate the survival and progression of ccRCC. Although there are still quite a few challenges remaining to be solved, the role of lncRNAs in clinical practice is a new hot spot. Multiple scholars have tried to use SNHG3 as a biomarker and therapeutic target for the diagnosis, treatment and monitoring of tumors. As mentioned earlier, many studies have shown that compared to normal tissues, tumor tissues have increased abnormally expressed SNHG3, which may be used as potential biomarkers for tumor diagnosis. 
SNHG3 as a potential therapeutic tool in cancer treatment It has been proven that abnormal small ncRNA expression levels in tumor cells can be used as effective drug targets for the treatment of tumors [85]. A wide range of ncRNAs have been discovered, namely, microRNAs (miRNAs), small interfering RNAs (siRNAs), and piwi-interacting RNAs (piRNAs). After these small RNAs (sRNAs) are incorporated into miRNA or siRNA-induced silencing complex (RISC), they participate in gene silencing in cells primarily through RNAi interference (RNAi) mechanisms $[86,87]$. What's more, antisense oligonucleotides (ASOs) are short single-stranded nucleic acid sequences, which form a stable hybrid with its target mRNA, thereby interfering with its processing or translation [88]. Although small ncRNAs have proven to be promising in vitro effective therapeutic drugs, it is very difficult to deliver these nucleic acid drugs into cells, and the low bioavailability of these nucleic acid drugs in vivo remains a major challenge. Therefore, ncRNA needs to be transported to the target tissue by a suitable carrier. Various small ncRNA vectors or systems have been proposed and widely explored for this purpose, including nanoparticles, ncRNA modification, and oncolytic adenovirus strategies. Nanoparticlebased small ncRNA vectors are the most common strategy [89]. SNHG3 is located in the nucleus and cytoplasm and requires a carrier to transport antisense RNAs or siRNAs into the cell to function [90]. Therefore, through continuous progress of antisense RNA or siRNA and ncRNA vectors, direct silencing of SNHG3 has become an important and powerful new target for tumor treatment.

\section{Conclusions and future perspectives}

Many studies have shown that lncRNA SNHG3 is overexpressed in most tumors, including breast cancer, osteosarcoma, glioma, laryngeal cancer, gastric cancer, colorectal cancer, renal cell carcinoma, hepatocellular carcinoma, lung cancer, acute myeloid leukemia, and epithelial ovarian cancer. Aberrant expression of SNHG3 is significantly associated with clinical features, such as

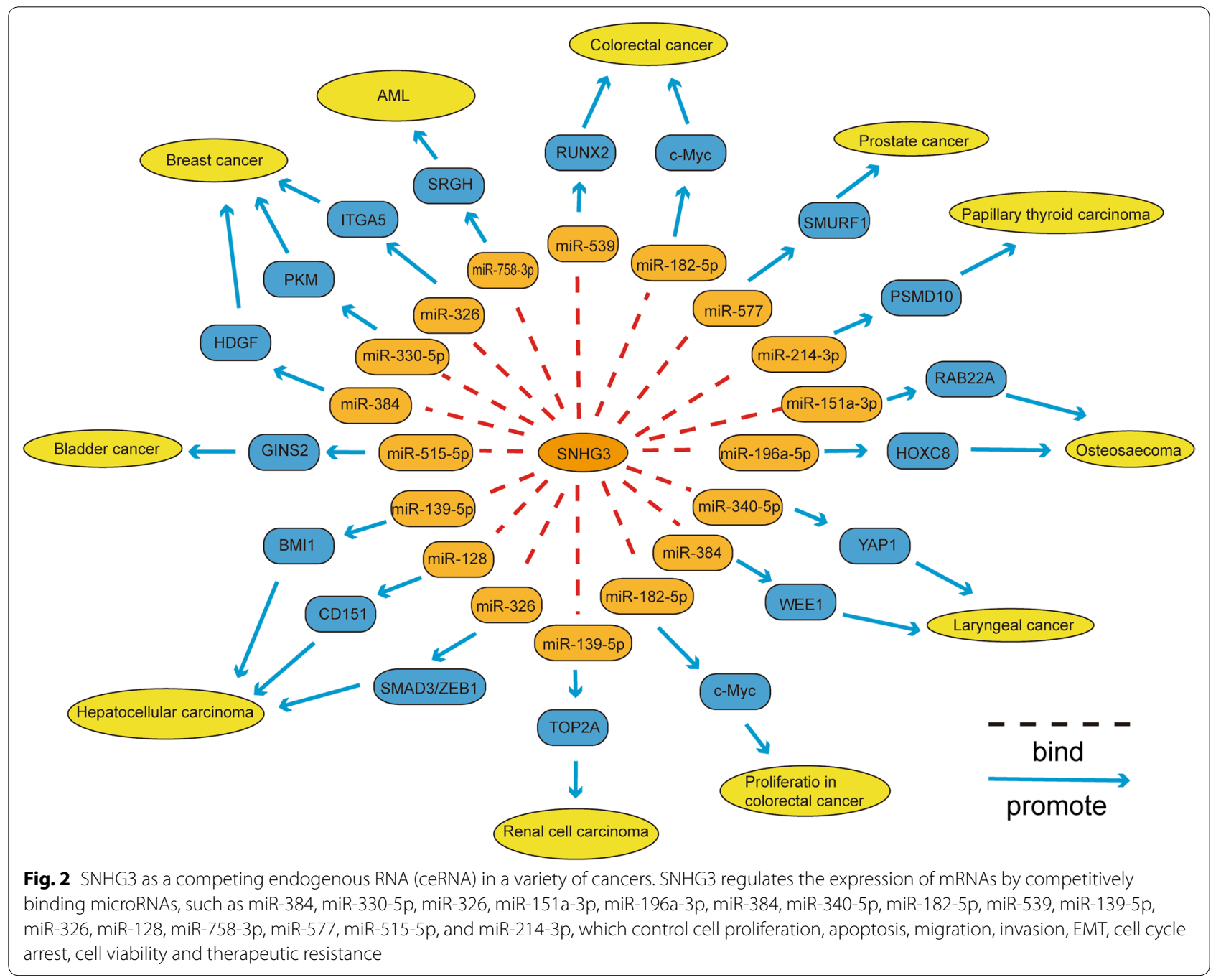


tumor size, TNM stage, lymph node metastasis, and overall patient survival. At the same time, abnormal expression of SNHG3 plays an important role in tumor biology in processes such as proliferation, migration, invasion and attenuation of apoptosis in tumor cells. In addition, a growing number of studies have shown that SNHG3, a ceRNA, competes with endogenous miRNA for binding, thereby inhibiting downstream genes of these miRNAs or regulating the development of tumor cells through classical pathways (Fig. 2). Therefore, SNHG3 will likely serve as a new target for tumor diagnosis and as a prognostic biomarker or a target for treating tumors. Although the molecular mechanisms of SNHG3 in many tumors have been studied, many questions remain. In addition, the precise molecular mechanism of SNHG3 requires further study, which will help in applying the results of SNHG3 research to clinical diagnosis and treatment of cancer.

\section{Abbreviations}

IncRNAs: Long noncoding RNAs; ceRNA: Competitive endogenous RNA; miRNAs: MicroRNAs; ncRNA: Noncoding RNAs; SNHG3: The small nuclear protein RNA host gene 3; AID: Activation-induced cytidine deaminase; mESC: Mouse embryonic stem cell; SnoRNAs: Small nucleolar RNAs; SNHG: Small nucleolar RNA host gene; HPA: Human Protein Atlas; TNM: Tumor node metastasis; ER: Estrogen receptor; Her-2: Human epidermal growth factor receptor 2; HDGF: Hepatoma-derived growth factor; PKM: Pyruvate kinase M1/M2; TNBC: Triple-negative breast cancer; ITGA5: Integrin a5; RAB22A: Ras-related proteins 22a; HOXC8: Homeobox c8; KLF2: Kruppel like factor 2; P21: P21 protein; LC: Laryngeal cancer; MMP2: Matrix metalloproteinase-2; MMP9: Matrix metalloproteinase-9; WEE1: Wee1-like protein kinase; YAP1:Yes-associated protein 1; GC: Gastric cancer; Hp: Helicobacter pylori; EZH2: Enhancer of Zeste Homolog 2; MED18: Mediator subunit18; CRC: Colorectal cancer; RUNX2: Runt-related transcription factor 2; RCC: Renal cell carcinoma; TOP2A: The expression of topoisomerase Ila; HCC: Hepatocellular carcinoma; OS: Overall survival; BMI1: B lymphoma Mo-MLV insertion region 1; EMT: Epithelial-mesenchymal transition; SMAD3: Sma and Mad Related Family 3; ZEB1: Zinc finger E-box binding homeobox 1; NSCLC: Non-small-cell lung cancer; E2F1: E2F transcription factor 1; TGF- $\beta$ : Transforming growth factor- $\beta$; JAK2: Janus kinase 2; STAT3: Signal transducer and activator of transcription 3; AML: Acute myeloid leukemia; SRGN: Serglycin; DNB: Dynamic Network Biomarkers; CML: Chronic myeloid leukemia; EOC: Epithelial ovarian cancer; OC: Ovarian cancer; FIGO: International federation of gynecology and obstetrics; GSK3B: Glycogen synthase kinase 3 beta; EIF4A3: Eukaryotic translation initiation factor 4A3; PPPM: Parents' Postoperative Pain Measure; SMURF1: Smad Ubiquitin Regulatory Factor 1; GINS2: GINS complex subunit 2; PSMD10: Proteasome 265 subunit non-ATPase 10; PTC: Papillary thyroid carcinoma; OSCC: Oral squamous cell carcinoma; ELAVL1: ELAV-like RNA-binding protein 1; NFYC: Nuclear transcription factor Y subunit gamma; ccRCC: Clear cell renal cell carcinoma; siRNAs: Small interfering RNA; piRNAs: Piwi-interacting RNA; RISC: SiRNA-induced silencing complex; RNAi: RNAi interference; ASOs: Antisense oligonucleotides.
\end{abstract}

\section{Acknowledgements}

Not applicable.

\begin{abstract}
Authors' contributions
JShao and JSun conceived the study and participated in the study design, performance, coordination and manuscript writing. BX, JM, WJ, ZB and JJ performed the literature review and graphics production. JShao and JSun revised the manuscript. All authors reviewed the final manuscript. All authors read and approved the final manuscript.
\end{abstract}

\section{Funding}

This work was supported by the National Natural Science Foundation of China (No. 8150215), Natural Science Foundation of Jiangsu Province (Nos.
BK20150004, BK20161140), Jiangsu Young Medical Talents (No. QNRC2016190), and Medical Talents of Wuxi People's Hospital (No. RKA201814).

\section{Availability of data and materials}

All data are included in the article.

\section{Ethics approval and consent to participate}

Not applicable.

\section{Consent for publication \\ Not applicable.}

\section{Competing interests}

The authors declare that they have no competing interests.

\section{Author details}

${ }^{1}$ Department of Neurosurgery, Wuxi People's Hospital Affiliated to Nanjing Medical University, No. 299 Qing Yang Road, Wuxi 214023, Jiangsu, China.

${ }^{2}$ Department of Oncology, Wuxi People's Hospital Affiliated to Nanjing Medical University, Wuxi, P. R. China.

Received: 31 July 2020 Accepted: 15 October 2020

Published online: 04 November 2020

\section{References}

1. Bray F, Ferlay J, Soerjomataram I, Siegel RL, Torre LA, Jemal A. Global cancer statistics 2018: GLOBOCAN estimates of incidence and mortality worldwide for 36 cancers in 185 countries. CA Cancer J Clin. 2018;68(6):394-424.

2. Torre LA, Bray F, Siegel RL, Ferlay J, Lortet-Tieulent J, Jemal A. Global cancer statistics, 2012. CA Cancer J Clin. 2015;65(2):87-108.

3. Allemani C, Matsuda T, Di Carlo V, Harewood R, Matz M, Niksic M, Bonaventure A, Valkov M, Johnson CJ, Esteve J, et al. Global surveillance of trends in cancer survival 2000-14 (CONCORD-3): analysis of individual records for 37513025 patients diagnosed with one of 18 cancers from 322 population-based registries in 71 countries. Lancet. 2018;391(10125):1023-75.

4. Kopp F, Mendell JT. Functional classification and experimental dissection of long noncoding RNAs. Cell. 2018;172(3):393-407.

5. Sullenger BA, Nair S. From the RNA world to the clinic. Science. 2016;352(6292):1417-20.

6. Yao RW, Wang Y, Chen LL. Cellular functions of long noncoding RNAs. Nat Cell Biol. 2019;21(5):542-51.

7. St Laurent G, Wahlestedt C, Kapranov P. The landscape of long noncoding RNA classification. Trends Genet. 2015;31(5):239-51.

8. Esteller M. Non-coding RNAs in human disease. Nat Rev Genet. 2011;12(12):861-74.

9. Beermann J, Piccoli MT, Viereck J, Thum T. Non-coding RNAs in development and disease: background, mechanisms, and therapeutic approaches. Physiol Rev. 2016;96(4):1297-325.

10. Fang Y, Fullwood MJ. Roles, functions, and mechanisms of long noncoding RNAs in cancer. Genom Proteom Bioinform. 2016;14(1):42-54.

11. Ransohoff JD, Wei Y, Khavari PA. The functions and unique features of long intergenic non-coding RNA. Nat Rev Mol Cell Biol. 2018;19(3):143-57.

12. Anfossi S, Babayan A, Pantel K, Calin GA. Clinical utility of circulating non-coding RNAs — an update. Nat Rev Clin Oncol. 2018;15(9):541-63.

13. Poller W, Dimmeler S, Heymans S, Zeller T, Haas J, Karakas M, Leistner DM, Jakob P, Nakagawa S, Blankenberg S, et al. Non-coding RNAs in cardiovascular diseases: diagnostic and therapeutic perspectives. Eur Heart J. 2018;39(29):2704-16.

14. Skalska L, Beltran-Nebot M, Ule J, Jenner RG. Regulatory feedback from nascent RNA to chromatin and transcription. Nat Rev Mol Cell Biol. 2017;18(5):331-7.

15. Esteller M, Pandolfi PP. The epitranscriptome of noncoding RNAs in cancer. Cancer Discov. 2017;7(4):359-68.

16. Anastasiadou E, Jacob LS, Slack FJ. Non-coding RNA networks in cancer. Nat Rev Cancer. 2018;18(1):5-18. 
17. Schmitt AM, Chang HY. Long noncoding RNAs in cancer pathways. Cancer Cell. 2016;29(4):452-63.

18. Matsui M, Corey DR. Non-coding RNAs as drug targets. Nat Rev Drug Discov. 2017;16(3):167-79.

19. Kato L, Begum NA, Burroughs AM, Doi T, Kawai J, Daub CO, Kawaguchi T, Matsuda F, Hayashizaki Y, Honjo T. Nonimmunoglobulin target loci of activation-induced cytidine deaminase (AID) share unique features with immunoglobulin genes. Proc Natl Acad Sci USA. 2012:109(7):2479-84

20. Lu W, Yu J, Shi F, Zhang J, Huang R, Yin S, Songyang Z, Huang J. The long non-coding RNA Snhg3 is essential for mouse embryonic stem cell selfrenewal and pluripotency. Stem Cell Res Ther. 2019;10(1):157.

21. Liang J, Wen J, Huang Z, Chen XP, Zhang BX, Chu L. Small nucleolar RNAs: insight into their function in cancer. Front Oncol. 2019;9:587.

22. Gong J, Li Y, Liu CJ, Xiang Y, Li C, Ye Y, Zhang Z, Hawke DH, Park PK, Diao $\mathrm{L}$, et al. A pan-cancer analysis of the expression and clinical relevance of small nucleolar RNAs in human cancer. Cell Rep. 2017;21(7):1968-81.

23. Xuan Y, Wang Y. Long non-coding RNA SNHG3 promotes progression of gastric cancer by regulating neighboring MED18 gene methylation. Cell Death Dis. 2019:10(10):694.

24. Fei F, He Y, He S, He Z, Wang Y, Wu G, Li M. LncRNA SNHG3 enhances the malignant progress of glioma through silencing KLF2 and p21. Biosci Rep. 2018. https://doi.org/10.1042/BSR20180420.

25. Zheng S, Jiang F, Ge D, Tang J, Chen H, Yang J, Yao Y, Yan J, Qiu J, Yin Z, et al. LncRNA SNHG3/miRNA-151a-3p/RAB22A axis regulates invasion and migration of osteosarcoma. Biomed Pharmacother. 2019;112:108695.

26. Li N, Zhan X. Anti-parasite drug ivermectin can suppress ovarian cancer by regulating IncRNA-EIF4A3-mRNA axes. EPMA J. 2020;11 (2):289-309.

27. DeSantis CE, Ma J, Goding Sauer A, Newman LA, Jemal A. Breast cancer statistics, 2017, racial disparity in mortality by state. CA Cancer J Clin. 2017;67(6):439-48.

28. Iqbal J, Ginsburg O, Rochon PA, Sun P, Narod SA. Differences in breast cancer stage at diagnosis and cancer-specific survival by race and ethnicity in the United States. JAMA. 2015;313(2):165-73.

29. Hanahan D, Weinberg RA. Hallmarks of cancer: the next generation. Cell. 2011;144(5):646-74.

30. Ward EM, DeSantis CE, Lin CC, Kramer JL, Jemal A, Kohler B, Brawley OW, Gansler T. Cancer statistics: breast cancer in situ. CA Cancer J Clin. 2015;65(6):481-95.

31. Ma Q, Qi X, Lin X, Li L, Chen L, Hu W. LncRNA SNHG3 promotes cell proliferation and invasion through the miR-384/hepatoma-derived growth factor axis in breast cancer. Hum Cell. 2020;33(1):232-42.

32. LiY, Zhao Z, Liu W, Li X. SNHG3 functions as miRNA sponge to promote breast cancer cells growth through the metabolic reprogramming. Appl Biochem Biotechnol. 2020;191(3):1084-99.

33. Wang P, Liu GZ, Wang JF, Du YY. SNHG3 silencing suppresses the malignant development of triple-negative breast cancer cells by regulating miRNA-326/integrin alpha5 axis and inactivating Vav2/Rac1 signaling pathway. Eur Rev Med Pharmacol Sci. 2020;24(10):5481-92.

34. Taherian-Esfahani Z, Taheri M, Dashti S, Kholghi-Oskooei V, Geranpayeh $\mathrm{L}$, Ghafouri-Fard S. Assessment of the expression pattern of mTOR-associated IncRNAs and their genomic variants in the patients with breast cancer. J Cell Physiol. 2019;234(12):22044-56.

35. Gianferante DM, Mirabello L, Savage SA. Germline and somatic genetics of osteosarcoma-connecting aetiology, biology and therapy. Nat Rev Endocrinol. 2017;13(8):480-91.

36. Harrison DJ, Geller DS, Gill JD, Lewis VO, Gorlick R. Current and future therapeutic approaches for osteosarcoma. Expert Rev Anticancer Ther. 2018;18(1):39-50.

37. Li Z, Dou P, Liu T, He S. Application of long noncoding RNAs in osteosarcoma: biomarkers and therapeutic targets. Cell Physiol Biochem. 2017:42(4):1407-19.

38. Chen J, Wu Z, Zhang Y. LncRNA SNHG3 promotes cell growth by sponging miR-196a-5p and indicates the poor survival in osteosarcoma. Int J Immunopathol Pharmacol. 2019;33:2058738418820743.

39. Chen R, Smith-Cohn M, Cohen AL, Colman H. Glioma subclassifications and their clinical significance. Neurotherapeutics. 2017;14(2):284-97.

40. Peng Z, Liu C, Wu M. New insights into long noncoding RNAs and their roles in glioma. Mol Cancer. 2018;17(1):61.

41. Cohen EE, LaMonte SJ, Erb NL, Beckman KL, Sadeghi N, Hutcheson KA, Stubblefield MD, Abbott DM, Fisher PS, Stein KD, et al. American cancer society head and neck cancer survivorship care guideline. CA Cancer J Clin. 2016;66(3):203-39.

42. Forastiere AA, Goepfert H, Maor M, Pajak TF, Weber R, Morrison W, Glisson B, Trotti A, Ridge JA, Chao C, et al. Concurrent chemotherapy and radiotherapy for organ preservation in advanced laryngeal cancer. N Engl J Med. 2003;349(22):2091-8.

43. Leemans CR, Snijders PJF, Brakenhoff RH. The molecular landscape of head and neck cancer. Nat Rev Cancer. 2018;18(5):269-82.

44. Wang L, Su K, Wu H, Li J, Song D. LncRNA SNHG3 regulates laryngeal carcinoma proliferation and migration by modulating the miR-384/WEE1 axis. Life Sci. 2019;232:116597.

45. Kang R, Yao DF, Xu GZ, Zhou YH. The knockdown of SNHG3 inhibits the progression of laryngeal squamous cell carcinoma by miR-340-5p/YAP1 axis and Wnt/beta-catenin pathway. Neoplasma. 2020;67(5):1094-105.

46. Siegel RL, Miller KD, Jemal A. Cancer statistics, 2019. CA Cancer J Clin. 2019;69(1):7-34.

47. Choi IJ, Kook MC, Kim YI, Cho SJ, Lee JY, Kim CG, Park B, Nam BH. Helicobacter pylori therapy for the prevention of metachronous gastric cancer. N Engl J Med. 2018;378(12):1085-95.

48. Lee YC, Chiang TH, Chou CK, Tu YK, Liao WC, Wu MS, Graham DY. Association between Helicobacter pylori eradication and gastric cancer incidence: a systematic review and meta-analysis. Gastroenterology. 2016;150(5):1113.e1115-1124.e1115.

49. Bonelli P, Borrelli A, Tuccillo FM, Silvestro L, Palaia R, Buonaguro FM. Precision medicine in gastric cancer. World J Gastrointest Oncol. 2019;11(10):804-29.

50. Quinn JJ, Chang HY. Unique features of long non-coding RNA biogenesis and function. Nat Rev Genet. 2016;17(1):47-62.

51. Miller KD, Siegel RL, Lin CC, Mariotto AB, Kramer JL, Rowland JH, Stein KD, Alteri R, Jemal A. Cancer treatment and survivorship statistics, 2016. CA Cancer J Clin. 2016;66(4):271-89.

52. Stiegelbauer V, Vychytilova-Faltejskova P, Karbiener M, Pehserl AM, Reicher A, Resel M, Heitzer E, Ivan C, Bullock M, Ling H, et al. miR-196b-5p regulates colorectal cancer cell migration and metastases through interaction with HOXB7 and GALNT5. Clin Cancer Res. 2017;23(17):5255-66.

53. Huang W, Tian Y, Dong S, Cha Y, Li J, Guo X, Yuan X. The long noncoding RNA SNHG3 functions as a competing endogenous RNA to promote malignant development of colorectal cancer. Oncol Rep. 2017;38(3):1402-10

54. Dacheng W, Songhe L, Weidong J, Shutao Z, Jingjing L, Jiaming Z. LncRNA SNHG3 promotes the growth and metastasis of colorectal cancer by regulating miR-539/RUNX2 axis. Biomed Pharmacother. 2020;125:110039.

55. Li JK, Chen C, Liu JY, Shi JZ, Liu SP, Liu B, Wu DS, Fang ZY, Bao Y, Jiang MM, et al. Long noncoding RNA MRCCAT1 promotes metastasis of clear cell renal cell carcinoma via inhibiting NPR3 and activating P38-MAPK signaling. Mol Cancer. 2017;16(1):111.

56. Motzer RJ, Tannir NM, McDermott DF, Aren Frontera O, Melichar B, Choueiri TK, Plimack ER, Barthelemy P, Porta C, George S, et al. Nivolumab plus ipilimumab versus sunitinib in advanced renal-cell carcinoma. N Engl J Med. 2018;378(14):1277-90.

57. Zhang C, Qu Y, Xiao H, Xiao W, Liu J, Gao Y, Li M, Liu J. LncRNA SNHG3 promotes clear cell renal cell carcinoma proliferation and migration by upregulating TOP2A. Exp Cell Res. 2019;384(1):111595.

58. Kulik L, El-Serag HB. Epidemiology and management of hepatocellular carcinoma. Gastroenterology. 2019;156(2):477.e471-491.e471.

59. Qiu L, Tang Q, Li G, Chen K. Long non-coding RNAs as biomarkers and therapeutic targets: recent insights into hepatocellular carcinoma. Life Sci. 2017;191:273-82.

60. Wu J, Liu L, Jin H, Li Q, Wang S, Peng B. LncSNHG3/miR-139-5p/BMl1 axis regulates proliferation, migration, and invasion in hepatocellular carcinoma. Onco Targets Ther. 2019;12:6623-38.

61. Zhao Q, Wu C, Wang J, Li X, Fan Y, Gao S, Wang K. LncRNA SNHG3 promotes hepatocellular tumorigenesis by targeting miR-326. Tohoku J Exp Med. 2019;249(1):43-56.

62. Zhang PF, Wang F, Wu J, Wu Y, Huang W, Liu D, Huang XY, Zhang XM, Ke AW. LncRNA SNHG3 induces EMT and sorafenib resistance by modulating the miR-128/CD151 pathway in hepatocellular carcinoma. J Cell Physiol. 2019;234(3):2788-94 
63. Zhang T, Cao C, Wu D, Liu L. SNHG3 correlates with malignant status and poor prognosis in hepatocellular carcinoma. Tumour Biol. 2016;37(2):2379-85.

64. Liu J, Li W, Zhang J, Ma Z, Wu X, Tang L. Identification of key genes and long non-coding RNA associated ceRNA networks in hepatocellular carcinoma. PeerJ. 2019;7:e8021.

65. Zhang R, Xia Y, Wang Z, Zheng J, Chen Y, Li X, Wang Y, Ming H. Serum long non coding RNA MALAT-1 protected by exosomes is up-regulated and promotes cell proliferation and migration in non-small cell lung cancer. Biochem Biophys Res Commun. 2017;490(2):406-14.

66. Shi J, Li J, Yang S, Hu X, Chen J, Feng J, Shi T, He Y, Mei Z, He W, et al. LncRNA SNHG3 is activated by E2F1 and promotes proliferation and migration of non-small-cell lung cancer cells through activating TGF-beta pathway and IL-6/JAK2/STAT3 pathway. J Cell Physiol. 2020;235(3):2891-900

67. Liu L, Ni J, He X. Upregulation of the long noncoding RNA SNHG3 promotes lung adenocarcinoma proliferation. Dis Mark. 2018;2018:5736716.

68. Arber DA, Orazi A, Hasserjian R, Thiele J, Borowitz MJ, Le Beau MM, Bloomfield CD, Cazzola M, Vardiman JW. The 2016 revision to the World Health Organization classification of myeloid neoplasms and acute leukemia. Blood. 2016;127(20):2391-405

69. Marcucci G, Haferlach T, Dohner H. Molecular genetics of adult acute myeloid leukemia: prognostic and therapeutic implications. J Clin Oncol. 2011:29(5):475-86.

70. Peng $L$, Zhang $Y$, Xin $H$. IncRNA SNHG3 facilitates acute myeloid leukemia cell growth via the regulation of miR-758-3p/SRGN axis. J Cell Biochem. 2020;121(2):1023-31.

71. Xu J, Wu M, Sun Y, Zhao H, Wang Y, Gao J. Identifying dysregulated IncRNA-associated ceRNA Network biomarkers in CML based on dynamical network biomarkers. Biomed Res Int. 2020;2020:5189549.

72. Lheureux S, Gourley C, Vergote I, Oza AM. Epithelial ovarian cancer. Lancet. 2019;393(10177):1240-53.

73. Li N, Zhan X, Zhan X. The IncRNA SNHG3 regulates energy metabolism of ovarian cancer by an analysis of mitochondrial proteomes. Gynecol Oncol. 2018;150(2):343-54.

74. Hong L, Chen W, Wu D, Wang Y. Upregulation of SNHG3 expression associated with poor prognosis and enhances malignant progression of ovarian cancer. Cancer Biomark. 2018;22(3):367-74.

75. Fahiminiya S, de Kock L, Foulkes WD. Biologic and clinical perspectives on thyroid cancer. N Engl J Med. 2016;375(23):2306-7.

76. Sui G, Zhang B, Fei D, Wang H, Guo F, Luo Q. The IncRNA SNHG3 accelerates papillary thyroid carcinoma progression via the miR-214-3p/PSMD10 axis. J Cell Physiol. 2020;235(10):6615-24.

77. Duan Y, Wang Z, Xu L, Sun L, Song H, Yin H, He F. IncRNA SNHG3 acts as a novel tumor suppressor and regulates tumor proliferation and metastasis via AKT/mTOR/ERK pathway in papillary thyroid carcinoma. J Cancer. 2020;11(12):3492-501.
78. Li T, Xing Y, Yang F, Sun Y, Zhang S, Wang Q, Zhang W. LncRNA SNHG3 sponges miR-577 to up-regulate SMURF1 expression in prostate cancer. Cancer Med. 2020;9(11):3852-62.

79. Dai G, Huang C, Yang J, Jin L, Fu K, Yuan F, Zhu J, Xue B. LncRNA SNHG3 promotes bladder cancer proliferation and metastasis through miR515-5p/GINS2 axis. J Cell Mol Med. 2020;24(16):9231-43.

80. Liu Z, Tao H. Small nucleolar RNA host gene 3 facilitates cell proliferation and migration in oral squamous cell carcinoma via targeting nuclear transcription factor Y subunit gamma. J Cell Biochem. 2020;121(3):2150-8.

81. Ye B, Shi J, Kang H, Oyebamiji O, Hill D, Yu H, Ness S, Ye F, Ping J, He J, et al. Advancing pan-cancer gene expression survial analysis by inclusion of non-coding RNA. RNA Biol. 2020;17(11):1666-73.

82. Lorenzi L, Avila Cobos F, Decock A, Everaert C, Helsmoortel H, Lefever S, Verboom K, Volders PJ, Speleman F, Vandesompele J, et al. Long noncoding RNA expression profiling in cancer: challenges and opportunities. Genes Chromosomes Cancer. 2019;58(4):191-9.

83. Hao S, Yao L, Huang J, He H, Yang F, Di Y, Jin C, Fu D. Genome-wide analysis identified a number of dysregulated long noncoding RNA (IncRNA) in human pancreatic ductal adenocarcinoma. Technol Cancer Res Treat. 2018;17:1533034617748429.

84. Yang W, Zhang K, Li L, Ma K, Hong B, Gong Y, Gong K. Discovery and validation of the prognostic value of the IncRNAs encoding snoRNAs in patients with clear cell renal cell carcinoma. Aging. 2020;12(5):4424-44.

85. To KK, Tong CW, Wu M, Cho WC. MicroRNAs in the prognosis and therapy of colorectal cancer: from bench to bedside. World J Gastroenterol. 2018;24(27):2949-73.

86. Snead NM, Rossi JJ. Biogenesis and function of endogenous and exogenous siRNAs. Wiley Interdiscip Rev RNA. 2010;1(1):117-31.

87. Petrek H, Batra N, Ho PY, Tu MJ, Yu AM. Bioengineering of a single long noncoding RNA molecule that carries multiple small RNAs. Appl Microbiol Biotechnol. 2019;103(15):6107-17.

88. Quemener AM, Bachelot L, Forestier A, Donnou-Fournet E, Gilot D, Galibert MD. The powerful world of antisense oligonucleotides: from bench to bedside. Wiley Interdiscip Rev RNA. 2020;11(5):e1594.

89. Wei L, Sun J, Zhang N, Zheng Y, Wang X, Lv L, Liu J, Xu Y, Shen Y, Yang M. Noncoding RNAs in gastric cancer: implications for drug resistance. Mol Cancer. 2020;19(1):62.

90. Zimta AA, Tigu AB, Braicu C, Stefan C, lonescu C, Berindan-Neagoe I. An Emerging class of long non-coding RNA with oncogenic role arises from the snoRNA host genes. Front Oncol. 2020;10:389.

\section{Publisher's Note}

Springer Nature remains neutral with regard to jurisdictional claims in published maps and institutional affiliations.

Ready to submit your research? Choose BMC and benefit from

- fast, convenient online submission

- thorough peer review by experienced researchers in your field

- rapid publication on acceptance

- support for research data, including large and complex data types

- gold Open Access which fosters wider collaboration and increased citations

- maximum visibility for your research: over $100 \mathrm{M}$ website views per year

At BMC, research is always in progress.

Learn more biomedcentral.com/submissions 